\title{
The Number of Real Quadratic Fields Having Units of Negative Norm
}

Peter Stevenhagen

\section{CONTENTS}

1. Introduction

2. Preliminaries

3. A Heuristic Approach to the Negative Pell Equation

4. Proven Densities for the Negative Pell Equation Acknowledgements

References
We study the density of the set of real quadratic fields for which the norm of the fundamental unit equals -1 inside the set of real quadratic fields containing elements of norm -1 . A conjectural density is derived from a single heuristic assumption, and experimental data supporting this assumption are given. We finally discuss how close one can get to proving such conjectural densities.

\section{INTRODUCTION}

The main problem in this paper, although formulated and treated in terms of real quadratic number fields, is a very old problem that does not need anything in its formulation beyond ordinary integers. More precisely, we will be concerned with the solvability of the negative Pell equation

$$
x^{2}-d y^{2}=-1 \quad \text { with } x, y \in \mathbf{Z}
$$

for squarefree numbers $d \in \mathbf{Z}_{>1}$. The solvability of this equation was studied by Euler, who mistakenly attached the name of the English mathematician John Pell (1611-1685) to the related equation $x^{2}-d y^{2}=1$. The problem of finding nontrivial solutions to the Pell equation itself had already been studied by many mathematicians long before $\mathrm{Eu}-$ ler's time, and Fermat, who posed it as a challenge to the English mathematicians in 1657, knew that it was solvable for all nonsquare $d>1$. An excellent account of the long history of the equation can be found in [Weil 1984].

In contrast to the relatively straightforward answer in the case of the Pell equation itself, the solvability of the negative Pell equation we are dealing with here turns out to be a much more complicated 
phenomenon. From the various density conjectures on real quadratic fields that will be discussed in this paper, it is easy to derive the following asymptotic result for the solvability of the negative Pell equation.

Conjecture 1.2. The number of squarefree integers $d$ up to $X$ for which the negative Pell equation is solvable is asymptotically equal to $c X / \sqrt{\log X}$, where $c \approx .2697$ is the constant defined by

$$
c=\frac{3}{2 \pi}\left(1-\prod_{\substack{j \geq 1 \\ j \text { odd }}}\left(1-2^{-j}\right)\right) \prod_{\substack{p \text { prime } \\ p \equiv 1 \bmod 4}}\left(1-p^{-2}\right)^{1 / 2} .
$$

Equation (1.1) is solvable if and only if the real quadratic order $\mathbf{Z}[\sqrt{d}]$ contains units of norm -1 , so deciding its solvability is equivalent to the determination of the norm $N e_{d}$ of a fundamental unit $e_{d} \in \mathbf{Z}[\sqrt{d}]$. Since $d$ is assumed to be squarefree, $\mathbf{Z}[\sqrt{d}]$ is of index $f \leq 2$ in the maximal order $\mathcal{O}_{D}$ of discriminant $D=f^{2} d$ in $\mathbf{Q}(\sqrt{d})$, and its unit group $\mathbf{Z}[\sqrt{d}]^{*}=\langle-1\rangle \times\left\langle e_{d}\right\rangle$ has index 1 or 3 in $\mathcal{O}_{D}^{*}=\langle-1\rangle \times\left\langle\varepsilon_{D}\right\rangle$. It follows that the negative Pell equation is solvable for $d$ if and only if the fundamental unit $\varepsilon_{D}$ of $\mathbf{Q}(\sqrt{d})$ has norm -1 . Thus, the problem we are dealing with is essentially a problem on units in real quadratic fields.

The determination of $N \varepsilon_{D}$ is easily accomplished if $D$ has a prime factor $p$ that is congruent to 3 modulo 4 , since in that case (1.1) is not solvable modulo $p$ and one has $N\left(\varepsilon_{D}\right)=1$. If $D$ has no such prime factors, (1.1) is solvable in every completion of $\mathbf{Q}(\sqrt{d})$, and by the Hasse principle this implies that it is solvable in $\mathbf{Q}$, that is, $\mathbf{Q}(\sqrt{d})$ contains elements of norm -1 . In order to find out whether there exists an integral solution, one has to determine $N \varepsilon_{D}$, and this can be done by looking at the parity of the length of the period of the continued fraction expansion of $\sqrt{d}$. As is well known, $N \varepsilon_{D}=-1$ if and only if this period length is odd [Stark 1970, Theorem 7.26]. This is a beautiful criterion in the sense that it provides an answer for any given discriminant, but it has the disadvantage that it does not tell us whether we should view the solvability of the negative Pell equation as an exception or as a common occurrence. This is also the case for the algorithms in [Lagarias 1980; Morton 1979; Rédei 1953b], which start from reformulations of (1.1) in the spirit of our Lemma 2.1 and determine $N \varepsilon_{D}$ by computing 2-class groups or 2-Hilbert class fields.

In order to make the preceding statement more precise, we let $\mathcal{D}$ be the set of discriminants of real quadratic fields that are not divisible by any prime congruent to $3 \bmod 4$. It follows from [Rieger 1965, Satz 3] that the number of such discriminants up to $X$ is asymptotically equal to $c_{1} X / \sqrt{\log X}$, where $c_{1}$ has the value

$$
c_{1}=\frac{9}{8 \pi} \prod_{\substack{p \text { prime } \\ p \equiv 1 \bmod 4}}\left(1-p^{-2}\right)^{1 / 2} .
$$

When counting radicands $d$ up to $X$, one can use the same formula with $\frac{4}{3} c_{1}$ instead of $c_{1}$.

Now let $\mathcal{D}^{-} \subset \mathcal{D}$ be the set of discriminants $D$ of real quadratic fields for which the fundamental unit $\varepsilon_{D}$ has norm -1 . Then our problem comes down to finding the density of $\mathcal{D}^{-}$in $\mathcal{D}$. In other words, if $\mathcal{D}_{\leqslant X}$ is the subset of $\mathcal{D}$ consisting of discriminants no greater than $X$, and $\mathcal{D}_{\leqslant X}^{-}$is defined likewise, we should ideally do the following:

Problem 1.3. Decide whether the limit

$$
P=\lim _{X \rightarrow \infty} \frac{\# \mathcal{D}_{\leqslant x}^{-}}{\# \mathcal{D}_{\leqslant x}}
$$

exists and, if so, determine it.

As it stands, this is a basic but very hard problem, and to my knowledge it is not even known whether the liminf and the limsup of this expression are in the open interval $(0,1)$. Numerical experiments show this to be very plausible, and Nagell [1932, p. 5] seems to have been the first to conjecture that $P$ exists and is in $(0,1)$. For small values of $X$ the values of the quotient in Problem 1.3 are shown in Table 1.

It can be shown that certain infinite sets of discriminants are contained in $\mathcal{D}^{-}$or in $\mathcal{D}^{+}=\mathcal{D} \backslash \mathcal{D}^{-}$, 


\begin{tabular}{|c|c|c|}
\hline$X$ & $\# \mathcal{D}_{\leqslant X}$ & $P=\frac{\# \mathcal{D}_{\leqslant X}^{-}}{\# \mathcal{D}_{\leqslant X}}$ \\
\hline $10^{4}$ & 1138 & .860 \\
$10^{5}$ & 10210 & .832 \\
$10^{6}$ & 93422 & .816 \\
$10^{7}$ & 866200 & .799 \\
\hline
\end{tabular}

TABLE 1. Numerical values of the ratio in Problem 1.3.

but the density in $\mathcal{D}$ of such sets is always zero. A classical example of an infinite set in $\mathcal{D}^{-}$is the set of prime discriminants $p \in \mathcal{D}$; it follows that

$$
\# \mathcal{D}_{\leqslant X}^{-} \gg \frac{X}{\log X} \quad \text { for } X \rightarrow \infty .
$$

Dirichlet [1834] showed that all odd discriminants $D=p q \in \mathcal{D}$ that are products of two primes $p$ and $q$ whose Legendre symbol $\left(\frac{p}{q}\right)$ equals -1 are also in $\mathcal{D}^{-}$. Moreover, he showed that if $\left(\frac{p}{q}\right)$ equals 1 and the product of the biquadratic residue symbols $\left(\frac{p}{q}\right)_{4}\left(\frac{q}{p}\right)_{4}$ equals -1 , then $D=p q$ is an element of $\mathcal{D}^{+}$. This implies an asymptotic lower bound

$$
\# \mathcal{D}_{\leqslant X}^{+} \gg \frac{X \log \log X}{\log X},
$$

a result that was completely overlooked in [Nagell 1932].

Even though there is an extensive literature on the equation (1.1) in general and on the density problem 1.3 in particular, it seems that none of the authors dealing with it [Cremona and Odoni 1989; Gerth 1984; Hurrelbrink 1990; Morton 1982; Nagell 1932; R. V. Perlis 1990; Rédei 1936; Scholz $1935]$ proposes a conjectural value for the asymptotic ratio $P$. One of the things we will do in this paper is supply both theoretical and experimental evidence for the following conjecture.

Conjecture 1.4. The limit value $P$ exists and equals

$$
1-\prod_{\substack{j \geq 1 \\ j \text { odd }}}\left(1-2^{-j}\right)=.5805775582 \ldots
$$

The numerical data given above do not seem to strongly support the conjectured value, but this is misleading, as we will see in Section 3. More carefully assembled experimental data do support our conjecture, and we will explain why values of $X$ for which $P$ is close to .58 are beyond the reach of any computer.

In the next section, it will be shown that our conjectured value of $P$ is an irrational number. As it has no obvious reason to be algebraic I expect it to be transcendental, but I do not know how to prove this.

The parity of the discriminant plays no role in our heuristics, so the conjecture should hold for even and odd discriminants separately. Using the result of Rieger discussed above, it is then straightforward to derive Conjecture 1.2 from this conjecture.

A resolution of Conjecture 1.4 seems to be well beyond reach at the moment. All methods developed so far prove density results that apply only to sets of discriminants having a fixed number of prime factors. In fact, the heuristics in Section 3 will furnish a conjectural value $P_{t}$ for the probability that $N \varepsilon_{D}=-1$ when $D \in \mathcal{D}$ is a discriminant having $t$ distinct prime factors. Since the expected number of prime factors of $D$ tends to infinity with $D$, it seems reasonable to expect $P$ to equal $\lim _{t \rightarrow \infty} P_{t}$. The numerical observations we present below agree quite nicely with our heuristics, especially in the precise formulation to be given in Conjecture 3.4. In the final Section 4, we will see how close we can get to proving the conjectural values for $P_{t}$ to be correct. The results, which are mostly due to Rédei $[1935 b ; 1936 ; 1949$; $1953 \mathrm{a} ; 1935 \mathrm{~b}$ ] can be viewed as vast generalizations of Dirichlet's biquadratic criterion mentioned above.

\section{PRELIMINARIES}

As it is more natural to work with discriminants than with radicands of quadratic fields, we will further look at the discriminant $D$ only and say that the negative Pell equation is solvable for the discriminant $D$ if the equation $x^{2}-D y^{2}=-4$ has 
integral solutions. This is the same as saying that $N \varepsilon_{D}=-1$ or that the negative Pell equation (1.1) is solvable for the corresponding radicand $d$.

For a real quadratic discriminant $D$, we let $C=$ $C_{D}$ be the narrow class group of $K=\mathbf{Q}(\sqrt{D})$, that is, the group of all fractional $\mathcal{O}_{D}$-ideals modulo the subgroup of principal ideals $(x)=x \mathcal{O}_{D}$ generated by some element $x \in K^{*}$ of positive norm. The group $C$ maps surjectively to the ordinary class group $\mathrm{Cl}$ of $K$, and the kernel is generated by the class $F_{\infty}$ of the principal ideal $(\sqrt{D})$. It is clear that $F_{\infty}$ is an element of the 2-torsion subgroup $C[2]$ of $C$. More precisely, the order of $F_{\infty}$ in $C$ is 2 if and only if all units in $\mathcal{O}_{D}$ have positive norm, or, equivalently, if and only if $N \varepsilon_{D}=1$.

Using class field theory, we can identify $C$ with the Galois group of the narrow Hilbert class field $H$ of $K$ over $K$. By definition, $H$ is the maximal abelian extension of $K$ in which all finite primes are unramified. The factor group $\mathrm{Cl}$ of $C$ corresponds to the maximal real subfield $\mathrm{H}^{+}$of $H$, and $\operatorname{Gal}\left(H / H^{+}\right)$is a cyclic group of order at most two, generated by the Frobenius symbol at infinity $F_{\infty}$.

We obtain the following alternative ways to look at the solvability of the negative Pell equation.

Lemma 2.1. The negative Pell equation is solvable for a quadratic discriminant $D$ if and only if the following equivalent conditions hold:

(i) $C=\mathrm{Cl}$;

(ii) the Frobenius at infinity $F_{\infty}=[(\sqrt{D})] \in C[2]$ is the unit element;

(iii) the narrow Hilbert class field of $\mathbf{Q}(\sqrt{D})$ is real.

The heuristic approach in the next section will be based on criterion (ii), but it is criterion (iii) that will be used in all proofs in Section 4.

Since condition (i) is satisfied for the $p$-primary parts of $C$ and $\mathrm{Cl}$ for every odd prime $p$, we can always restrict our attention to the 2-primary part of $C$. In the same way, it suffices to look at the archimedean character of the narrow 2-Hilbert class field in (iii).
There is a part of the extension $H / K$, the genus field extension $G / K$ corresponding to the factor group $C / C^{2}$, that can be easily generated in terms of $D$. One obtains $G$ from $K$ by adjoining a square root of $(-1)^{(p-1) / 2} p$ for each odd prime divisor $p$ of $D$. The extension $G / \mathbf{Q}$ is the largest abelian subextension of $H / \mathbf{Q}$. Note that $G$ is real if and only if $D$ is in $\mathcal{D}$. Using criterion (iii), it follows once more that the negative Pell equation can only be solvable for $D \in \mathcal{D}$.

On the other hand, there is a part of the class group $C$ consisting of 2-torsion or ambiguous ideal classes that can also be generated easily. One can take the classes of the primes of $K$ that are ramified over $\mathbf{Q}$ as generators, and as we have

$$
\# C[2]=\#\left(C / C^{2}\right)=2^{t-1}
$$

for a discriminant having $t$ distinct prime factors, there is exactly one nontrivial relation between the classes of the ramified primes of $K$. For discriminants $D \in \mathcal{D}$, this relation is $F_{\infty}=1$ if and only if the negative Pell equation is solvable for $D$.

One can go a step further, following Rédei, and combine the knowledge of $C[2]$ and $C / C^{2}$ to give a description of the 4-rank $e_{4}(D)$ of $C$, i.e., the number of cyclic summands of $C$ having order divisible by 4 . One observes that the 4 -rank of $C$ is the dimension, over the field $\mathbf{F}_{2}$ of 2 elements, of the kernel of the natural map $C[2] \rightarrow C / C^{2}$ and looks at the explicit description of this map in terms of the prime divisors of $D$. The result is the following theorem of Rédei and Reichardt, valid for any quadratic discriminant $D$. For a proof one can read the original paper [Rédei and Reichardt 1934], do the exercises following [Borevich and Shafarevich 1966, Chapter 3] or consult [Stevenhagen a].

Proposition 2.2. Let $D=\prod_{i=1}^{t} d_{i}$ be the decomposition of a quadratic discriminant $D$ into prime power discriminants. Then the 4-rank of the narrow class group $\mathcal{C}_{D}$ equals

$$
e_{4}(D)=t-1-\operatorname{rank} M_{D}
$$


where $M_{D}=\left(\varepsilon_{i j}\right)_{i, j=1}^{t}$ is the matrix over $\mathbf{F}_{2}$ defined by $(-1)^{\varepsilon_{i j}}=\left(\frac{d_{i}}{d_{j}}\right)$ if $i \neq j$ and $\varepsilon_{j j}=\sum_{i \neq j} \varepsilon_{i j}$. Here we set $\left(\frac{x}{d_{j}}\right)=\left(\frac{x}{2}\right)=(-1)^{(x-1) / 4}$ if $d_{j}$ is even.

The matrix $M_{D}$ is called the Rédei matrix of the discriminant $D$. Since we can permute the discriminantal divisors $d_{i}$, it is only defined up to conjugation by a permutation matrix. We will assume from now on that $M_{D}$ is the matrix obtained by arranging the discriminantal divisors $d_{i}$ of $D$ in ascending order. Note that, for $D \in \mathcal{D}$, the $d_{i}$ are either primes congruent to 1 modulo 4 or equal to 8. In this case, we usually write $p_{i}$ for $d_{i}$.

Let $\psi_{D}: V=\mathbf{F}_{2}^{t} \rightarrow C[2]$ be the canonical surjection that maps the $i$-th basis vector in $V$ to the class in $C$ of the prime over $d_{i}$. Then the kernel ker $M_{D} \subset V$ of $M_{D}$ is the preimage $\psi_{D}^{-1}\left(C[2] \cap C^{2}\right)$ of the set of ambiguous ideal classes that lie in the principal genus. Its $\mathbf{F}_{2}$-dimension equals $e_{4}(D)+1$.

For $D \in \mathcal{D}$, the quadratic reciprocity law implies that the Rédei matrix $M_{D}$ is a symmetric matrix whose rows and columns add up to zero. Such a matrix $M_{D}$ is completely determined by its $(1,1)$ minor $M_{D}^{\prime}$, a symmetric $(n-1) \times(n-1)$ matrix over $\mathbf{F}_{2}$. Moreover, $\operatorname{rank} M_{D}=\operatorname{rank} M_{D}^{\prime}$.

We will need to count symmetric matrices over the field of two elements having a prescribed rank. The cardinality of our base field does not play any role, so we state the result for arbitrary finite fields.

Proposition 2.3. Let $n \geq 1$ be an integer and $q$ a prime power. Then there are exactly

$$
A_{n}(q)=q^{\left(\begin{array}{c}
n+1 \\
2
\end{array}\right)} \prod_{\substack{1 \leq k \leq n \\
k \text { odd }}}\left(1-q^{-k}\right)
$$

nonsingular symmetric $n \times n$ matrices over the field $\mathbf{F}_{q}$ of $q$ elements. The number of matrices of arbitrary rank $r=0, \ldots, n$ is $\left[\begin{array}{l}n \\ r\end{array}\right]_{q} A_{r}(q)$, where $\left[\begin{array}{l}n \\ r\end{array}\right]_{q}$ denotes the number of $r$-dimensional subspaces of a vector space of dimension $n$ over $\mathbf{F}_{q}$.

Proof. A completely elementary proof by induction on $n$ can be found in [MacWilliams 1969]. In order to see that the statement given there is identical to ours one needs the explicit value

$$
\left[\begin{array}{l}
n \\
r
\end{array}\right]_{q}=\frac{\prod_{i=1}^{n}\left(q^{i}-1\right)}{\prod_{i=1}^{r}\left(q^{i}-1\right) \prod_{i=1}^{n-r}\left(q^{i}-1\right)} .
$$

The first half of the proposition immediately implies the second half, as symmetric matrices correspond bijectively to symmetric bilinear forms, and giving a symmetric bilinear form of rank $r$ on $V=\mathbf{F}_{q}^{n}$ is equivalent to giving a subspace $W \subset V$ of dimension $n-r$ and a nondegenerate symmetric bilinear form of the factor space $V / W$. This remark also shows that the numbers $A_{n}(q)$ can be computed inductively from the relation

$$
\sum_{r=0}^{n}\left[\begin{array}{l}
n \\
r
\end{array}\right]_{q} A_{r}(q)=q^{\left(\begin{array}{c}
n+1 \\
2
\end{array}\right),}
$$

so it suffices to check that the given expression satisfies this relation. An elegant way of doing this is given in [Cremona and Odoni 1989].

Corollary 2.4. Among all $t \times t$ matrices that can be realized as the Rédei matrix $M_{D}$ of some discriminant $D \in \mathcal{D}$, the fraction of matrices having rank $t-1-e$ equals

$$
\alpha_{t}(e)=2^{-\left(\begin{array}{c}
e+1 \\
2
\end{array}\right)} \frac{\prod_{j=e+1}^{t-1}\left(1-2^{-j}\right)}{\prod_{j=1}^{(t-e-1) / 2}\left(1-2^{-2 j}\right)} .
$$

Proof. Rather than considering the Rédei matrices themselves, one looks at the corresponding $(1,1)$ minors. They range over the full set of symmetric $(t-1) \times(t-1)$ matrices over $\mathbf{F}_{2}$, since one can always find primes congruent to 1 modulo 4 that have prescribed Legendre symbols modulo a finite number of primes. One deduces that

$$
\alpha_{t}(e)=2^{-\left(\begin{array}{l}
t \\
2
\end{array}\right)}\left[\begin{array}{c}
t-1 \\
t-1-e
\end{array}\right]_{2} A_{t-1-e}(2) .
$$

The result follows upon substitution of the explicit values given in the proposition.

In order to interpret the numbers $\alpha_{t}(e)$ as densities of the kind we need, we still have to know that if $D$ ranges over the subset $\mathcal{D}_{t}$ of $\mathcal{D}$ consisting 
of those discriminants that have exactly $t$ distinct prime divisors, then the corresponding Rédei minor $M_{D}^{\prime}$ behaves like a random symmetric $(t-1) \times(t-1)$ matrix over $\mathbf{F}_{2}$ (with repect to the uniform distribution). This is equivalent to saying that the vector consisting of the $\left(\begin{array}{l}t \\ 2\end{array}\right)$ Legendre symbols $\left(\frac{p_{i}}{p_{j}}\right)$ of an element $D=p_{1} p_{2} \ldots p_{t}$ is uniformly distributed as a function on $\mathcal{D}_{t}$. The following result was obtained by Rédei [1939], but his proof is incorrect in its estimate of the error term. A complete proof was given more than fifty years later [Cremona and Odoni 1989]. An analogous result for the Rédei matrices of arbitrary quadratic discriminants has been proved and exploited by Gerth [1984].

Proposition 2.5. Let $S$ be a $t \times t$ matrix over $\mathbf{F}_{2}$ for which $\mathcal{D}_{S}=\left\{D \in \mathcal{D}: M_{D}=S\right\}$ is nonempty. Then

$$
\lim _{X \rightarrow \infty} \frac{\#\left(\mathcal{D}_{S}\right)_{\leqslant X}}{\#\left(\mathcal{D}_{t}\right)_{\leqslant x}}=2^{-\left(\begin{array}{c}
t \\
2
\end{array}\right)} .
$$

(Here, of course, $\left(\mathcal{D}_{S}\right)_{\leqslant X}$ is the set of $D \in D_{S}$ not exceeding $X$, and similarly for $\left(\mathcal{D}_{t}\right)_{\leqslant X}$.) Proposition 2.5 says that all symmetric $t \times t$ matrices with rows and columns adding up to zero occur with the same frequency as the Rédei matrix of discriminants $D \in \mathcal{D}_{t}$. It follows that $\alpha_{t}(e)$ is the natural density in $\mathcal{D}_{t}$ of the set of discriminants $D \in \mathcal{D}_{t}$ that give rise to a narrow class group of 4-rank $e_{4}(D)=e$. We set $\alpha_{t}(e)=0$ for $e \geq t$.

It is easy to see that each of the sets $\mathcal{D}_{t}$ has zero density in $\mathcal{D}$. In fact, a slight modification of [Hardy and Wright 1979, Theorem 437] shows that for $X \rightarrow \infty$ one has the asymptotic result

$$
\#\left(\mathcal{D}_{t}\right)_{\leqslant X} \sim \frac{2^{-t}}{(t-1) !} \frac{X(\log \log X)^{t-1}}{\log X} .
$$

It implies that the expected number of prime factors of a discriminant $D \in \mathcal{D}$ tends to infinity with $D$. More precisely, it can be shown [Hardy and Wright 1979, Theorem 430] that this expected number is asymptotically equal to $\log \log D$ for $D$ tending to infinity. It is therefore of interest to study the behavior of $\alpha_{t}(e)$ when $e$ is fixed and $t$ tends to infinity. Here we will encounter the infinite product

$$
\alpha=\prod_{j \text { odd }}\left(1-2^{-j}\right)=.41942244117951 \ldots
$$

occurring in Conjecture 1.4.

Proposition 2.8. Let e be a nonnegative integer and let $\alpha$ be as in (2.7). Then the limit value $\alpha_{\infty}(e)=$ $\lim _{t \rightarrow \infty} \alpha_{t}(e)$ equals

$$
\alpha_{\infty}(e)=\frac{\alpha}{\prod_{j=1}^{e}\left(2^{j}-1\right)} .
$$

Moreover, we have $\sum_{e=0}^{\infty} \alpha_{\infty}(e)=1$.

Proof. The value of $\alpha_{\infty}(e)$ follows immediately from Corollary 2.4. For the second statement, we have to show that we can interchange sum and limit in the left-hand side of the trivial identity

$$
\lim _{t \rightarrow \infty} \sum_{e=0}^{\infty} \alpha_{t}(e)=\lim _{t \rightarrow \infty} 1=1 .
$$

This follows from Lebesgue's theorem on dominated convergence if we interpret the sum as the integral over $\mathbf{Z}_{\geq 0}$ with respect to the counting measure and observe that the sequence of nonnegative functions $\left\{\alpha_{t}\right\}_{t=1}^{\infty}$ is uniformly bounded by an integrable function $F: \mathbf{Z}_{\geq 0} \rightarrow \mathbf{R}$. We can take

$$
F(e)=2^{-\left(\begin{array}{c}
e+1 \\
2
\end{array}\right)} \prod_{j \geq 1}\left(1-2^{-2 j}\right)^{-1} .
$$

Remark. The identity $\sum_{e=0}^{\infty} \alpha_{\infty}(e)=1$ in the preceding proposition can be proved directly, without the use of the values $\alpha_{t}(e)$. In our special case it comes down to showing the identity

$$
\begin{aligned}
1+1+\frac{1}{3}+\frac{1}{3 \cdot 7}+\frac{1}{3 \cdot 7 \cdot 15} & +\frac{1}{3 \cdot 7 \cdot 15 \cdot 31}+\cdots \\
& =\frac{2}{1} \cdot \frac{8}{7} \cdot \frac{32}{31} \cdot \frac{128}{127} \cdots,
\end{aligned}
$$

and in the case of a base field of arbitrary cardinality $q$ it would be 


$$
\begin{aligned}
1+\frac{1}{q-1}+\frac{1}{(q-1)\left(q^{2}-1\right)} & +\cdots \\
& =\prod_{j \text { odd }}\left(1-q^{-j}\right)^{-1} .
\end{aligned}
$$

This is a formal identity, and both sides converge in the complex plane whenever $|q|>1$. It is an example of a partition identity, since the right hand side is equal to

$$
\prod_{j \geq 1}\left(1+q^{-j}\right)=1+\sum_{n \geq 1} r(n) q^{-n}
$$

where $r(n)$ is the number of ways in which $n$ can be written as a sum of distinct positive integers. In order to prove such an identity, one expands the factors $\left(q^{i}-1\right)^{-1}$ in the left hand side in geometric series and rewrites this side as

$$
1+\sum_{k \geq 1} \sum_{i_{1}, i_{2}, \ldots, i_{k}>0} q^{-\left(i_{1}+2 i_{2}+\cdots+k i_{k}\right)}
$$

It now suffices to recall the standard identity

$r(n)=\sum_{k \geq 1} \#\left\{\left(i_{1}, i_{2}, \ldots, i_{k}\right) \in \mathbf{Z}_{>0}^{k}: \sum_{j=1}^{k} j i_{j}=n\right\}$,

which is immediately seen to hold if one writes the partitions of $n$ in the form

$n=\left(i_{1}+i_{2}+\cdots+i_{k}\right)+\left(i_{2}+i_{3}+\cdots+i_{k}\right)+\cdots+i_{k}$.

Tijdeman observed that the value of (2.9) is irrational for any integral value $q>1$. The proof of this fact is completely analogous to the standard irrationality proof of the number $e=\sum_{n \geq 0}(n !)^{-1}$, which is also the limit value of a series with rational terms in which the $n$-th term is a large integral multiple of the $(n+1)$-th term.

\section{A HEURISTIC APPROACH TO THE NEGATIVE PELL EQUATION}

We will first apply criterion (ii) of Lemma 2.1 to find a heuristic value for the probability that the negative Pell equation is solvable for an arbitrary discriminant in $\mathcal{D}_{t}$, that is, a conjectural value for the limit

$$
P_{t}=\lim _{X \rightarrow \infty} \frac{\#\left(\mathcal{D}_{t}^{-}\right)_{\leqslant X}}{\#\left(\mathcal{D}_{t}\right)_{\leqslant X}}
$$

when $t \in \mathbf{Z}_{>0}$ is fixed. Here $\mathcal{D}_{t}^{-}$stands for the set of $D \in \mathcal{D}_{t}$ for which the negative Pell equation is solvable, and $\left(\mathcal{D}_{t}^{-}\right)_{\leqslant x}$ for the subset of $\mathcal{D}_{t}$ with $D \leq X$. Note that the mere existence of these limits is already a highly nontrivial statement.

Since all prime discriminants in $\mathcal{D}$ are in $\mathcal{D}^{-}$, it is a theorem that $P_{1}$ exists and is equal to 1 . No other value of $P_{t}$ has so far been shown to exist.

In order to illustrate what happens for general $t$, we start with the special family of discriminants $D=8 p \in \mathcal{D}_{2}$ studied in [Stevenhagen 1993]. For $p \equiv 5 \bmod 8$, we have $\left(\frac{2}{p}\right)=-1$ and Rédei's result (Proposition 2.2) implies that $e_{4}(8 p)=0$. This means that the narrow 2-Hilbert class field coincides with the genus field $G=\mathbf{Q}(\sqrt{2}, \sqrt{p})$. In this case we have $N \varepsilon_{8 p}=-1$ by Lemma (2.1)(iii). In the other case, $p \equiv 1 \bmod 8$, the situation is more complicated, since Proposition 2.2 gives $e_{4}(8 p)=$ 1. We now look at the 2 -torsion subgroup of $C$, which is generated by the primes $\mathfrak{t}$ and $\mathfrak{p}$ lying over 2 and $p$. Exactly one of the three ideal classes $[t]$, $[\mathfrak{p}]$ and $[\mathfrak{t} \mathfrak{p}]$ is the trivial element in $C[2]$. Numerical investigation of all $p \equiv 1 \bmod 8$ under $10^{6}$ indicates that these three possibilities occur with the same frequency, even though the best thing one can prove [Stevenhagen 1993] is that each of the three cases occurs with frequency $\geq \frac{1}{4}$ when $p$ ranges over the primes $p \equiv 1 \bmod 8$. The negative Pell equation for $8 p$ is solvable if and only if $F_{\infty}=[\mathfrak{t p}] \in C$ is the trivial element, so a combination of the theorem for $p \equiv 5 \bmod 8$ and the numerical observation for $p \equiv 1 \bmod 8$ would imply that $N \varepsilon_{8 p}=-1$ for two out of three primes $p \equiv 1 \bmod 4$.

Apart from a few obvious changes, the argument just given remains correct when the discriminantal divisor 8 is replaced by an odd prime $q \equiv 1 \bmod 4$. One is therefore led to the conjectural value $P_{2}=\frac{2}{3}$ for the fraction of discriminants in $\mathcal{D}_{2}$ for which the negative Pell equation is solvable. 
The heuristic reasoning we gave can be adapted to discriminants with exactly $t$ prime divisors in the following way. Let

$$
\psi_{D}: V=\mathbf{F}_{2}^{t} \rightarrow C[2],
$$

for $D \in \mathcal{D}_{t}$, be the surjection described in the previous section. Then the kernel of $\psi_{D}$ is a onedimensional subspace $\mathbf{F}_{2} \cdot r_{D}$, where $r_{D} \in V$ can be thought of as the nontrivial relation between the canonical generators of $C[2]$. It is clear that $r_{D}$ is contained in the kernel

$$
\operatorname{ker} M_{D}=\psi_{D}^{-1}\left(C[2] \cap C^{2}\right) \subset V
$$

of the Rédei map. Let $u=(1)_{i=1}^{t} \in V$ be the element whose $\psi_{D}$-image is the Frobenius at infinity $F_{\infty} \in C[2]$. Then $u$ is also contained in $\operatorname{ker} M_{D}$. This follows from the fact that the genus field $G$ is real, but it can also be seen directly from the form of the Rédei matrix $M_{D}$, which has columns adding up to zero for $D \in \mathcal{D}$. We have $D \in \mathcal{D}^{-}$if and only if $r_{D}=u$, and the basis of the argument we gave for $D=8 p$ is that we suppose $r_{D}$ to be a random nonzero element in $\operatorname{ker} M_{D}$. This is an $\mathbf{F}_{2}$-vector space of dimension $e_{4}(D)+1$, so for $D$ ranging over $\mathcal{D}_{t}(e)$ we expect to have $N \varepsilon_{D}=-1$ in 1 out of $2^{e+1}-1$ cases. We can formulate this heuristic assumption more precisely in the following way.

Hypothesis 3.1. Let $t>0$ be given, and define $r_{D} \in$ $V=\mathbf{F}_{2}^{t}$ for $D \in \mathcal{D}_{t}$ as above. Suppose $S \in$ End $V$ is a symmetric $t \times t$ matrix for which

$$
\mathcal{D}_{S}=\left\{D \in \mathcal{D}: M_{D}=S\right\}
$$

is nonempty, and let $d$ be the dimension of $\operatorname{ker} S$.

Then

$$
\lim _{X \rightarrow \infty} \frac{\#\left\{D \in \mathcal{D}_{S}: D \leq X \text { and } r_{D}=v\right\}}{\#\left\{D \in \mathcal{D}_{S}: D \leq X\right\}}=\frac{1}{2^{d}-1}
$$

for every nonzero element $v \in \operatorname{ker} S$.

For fixed $t$, we write $\mathcal{D}_{t}$ as a disjoint union

$$
\mathcal{D}_{t}=\bigcup_{e=0}^{t-1} \mathcal{D}_{t}(e)
$$

where $\mathcal{D}_{t}(e)$ is the subset of $D \in \mathcal{D}_{t}$ that have $e_{4}(D)=e$. We have already seen that $\mathcal{D}_{t}(e)$ has natural density $\alpha_{t}(e)$ inside $\mathcal{D}_{t}$. The preceding hypothesis, when applied to the element $v=u=$ $(1)_{i=1}^{t} \in V$, implies that the density inside $\mathcal{D}_{t}(e)$ of the set of discriminants $D \in \mathcal{D}_{t}(e)$ for which the negative Pell equation is solvable is $\left(2^{e+1}-1\right)^{-1}$. Taking the union of these sets for $e=0,1, \ldots, t-1$, we arrive at the following heuristic value for $P_{t}$ :

Theorem 3.2. Suppose that Hypothesis 3.1 is satisfied. Then the set $\mathcal{D}_{t}^{-}$of $D \in \mathcal{D}_{t}$ for which the negative Pell equation is solvable has a natural density inside $\mathcal{D}_{t}$ equal to

$$
P_{t}=\sum_{e=0}^{t-1} \frac{\alpha_{t}(e)}{2^{e+1}-1}
$$

where the numbers $\alpha_{t}(e)$ are defined as in Corollary 2.4 .

If $D$ tends to infinity, the expected value of $t$ tends to infinity as $\log \log D$. It is therefore to be expected that if the natural density $P$ of $\mathcal{D}^{-}$in $\mathcal{D}$ exists, it is equal to $\lim _{t \rightarrow \infty} P_{t}$. This is how we obtain Conjecture 1.4. The computation of the limit is dealt with in the following lemma.

Lemma 3.3. For $t$ tending to infinity, the density $P_{t}$ of the preceding theorem tends to a limit value

$$
P_{\infty}=1-\prod_{j \text { odd }}\left(1-2^{-j}\right)=.5805775582 \ldots
$$

Proof. Using the limit values $\alpha_{\infty}(e)$ from Proposition 2.8 , one obtains

$$
\begin{aligned}
P_{\infty} & =\lim _{t \rightarrow \infty} \sum_{e=0}^{t-1} \frac{\alpha_{t}(e)}{2^{e+1}-1}=\sum_{e=0}^{\infty} \frac{\alpha_{\infty}(e)}{2^{e+1}-1} \\
& =\sum_{e=0}^{\infty} \frac{\alpha}{\prod_{j=1}^{e+1}\left(2^{j}-1\right)}=\sum_{e=1}^{\infty} \alpha_{\infty}(e) \\
& =1-\alpha_{\infty}(0)=1-\alpha
\end{aligned}
$$


The justification for interchanging sum and limit follows as in the proof of Proposition 2.8, since the functions

$$
e \mapsto \frac{\alpha_{t}(e)}{2^{e+1}-1}
$$

on $\mathbf{Z}_{\geq 0}$ are also bounded by the integrable function $F$ given there.

Since the predicted density of the set $\mathcal{D}_{t}^{-}(e)$ consisting of discriminants in $\mathcal{D}_{t}(e)$ for which the negative Pell equation is solvable inside $\mathcal{D}_{t}(e)$ does not depend on $t$, we can formulate our conjectured behavior more directly as follows.

Conjecture 3.4. For $e \geq 0$, let $\mathcal{D}(e)$ denote the subset of $\mathcal{D}$ consisting of discriminants having 4-rank $e$, and $\mathcal{D}^{-}(e)$ the set of $D \in \mathcal{D}(e)$ for which the negative Pell equation is solvable. Define $\alpha_{\infty}(e) \in$ $\mathbf{R}$ as in Proposition 2.8. Then, for every integer $e \geq 0$,

(i) the natural density of $\mathcal{D}^{-}(e)$ inside $\mathcal{D}(e)$ equals $\left(2^{e+1}-1\right)^{-1}$, and

(ii) the natural density of $\mathcal{D}(e)$ inside $\mathcal{D}$ equals $\alpha_{\infty}(e)$.

In particular, the natural density of $\mathcal{D}^{-}$inside $\mathcal{D}$ exists and is equal to

$$
P=\sum_{e=0}^{\infty} \frac{\alpha_{\infty}(e)}{2^{e+1}-1}=1-\alpha .
$$

Note that the final statement of the conjecture does indeed follow from (i) and (ii), even though we do not have a countably additive measure on $\mathcal{D}$. It is essential for this that the densities of the sets $\mathcal{D}(e)$ add up to $\sum_{e=0}^{\infty} \alpha_{\infty}(e)=1$.

As a result of the computation in Lemma 3.3, we have found that the conjectural density of $\mathcal{D}^{+}$ in $\mathcal{D}$ is equal to the probability for a symmetric matrix over $\mathbf{F}_{2}$ to be nonsingular when the size of the matrix tends to infinity. This raises the natural question whether there is a more direct heuristic reasoning leading to the result. No such reasoning is known to me.
A weaker form of the basic Hypothesis 3.1 of this section, which would have sufficed for our purposes, can be rephrased somewhat informally as the hypothesis that, for $D$ ranging over $\mathcal{D}$, the relation between the classes of the ramified primes behaves randomly when considered inside the 2torsion subgroup of the principal genus $C^{2}$. There is a certain theoretical motivation for looking at $C^{2}$ rather than $C$ itself. Namely, according to the Cohen-Lenstra heuristics for class groups of real quadratic fields [Cohen and Lenstra 1984], there is an expected behavior of the $p$-part of $C$ that works well in practice for odd $p$, but is provably wrong for $p=2$ because of the genus theory for these class groups. However, the heuristics do seem to work for all $p$ when one considers the principal genus $C^{2}$ instead of $C$. More precisely, Gerth [1984] has shown - and this is one of the rare cases where anything of this kind can be proved - that the distribution of the 2-rank of $C^{2}$ for fixed $t$ tends to a limit distribution that is predicted by Cohen and Lenstra when one lets $t$ tend to infinity.

For an idea of of the numerical behavior of the various constants discussed so far, see Table 2.

Although tables related to the negative Pell equation have been produced since the time of Euler, there does not seem to exist anything in the literature that can be used in numerically checking our conjectures. Existing tables [Thielmann 1926; Nagell 1932; Beach and Williams 1972] mostly tabulate the values of $d$ up to a given bound for which the negative Pell equation (1.1) is solvable. Usually, they include values of $d$ that are not squarefree and that we chose to disregard. Moreover, all even discriminants are listed as $D / 4$ and are therefore overrepresented in the tables. Even making allowances for all this, the fact that all tables up to the most recent I know of [Beach and Williams 1972] only include values of $D<10^{6}$ makes them almost worthless in numerically checking a conjecture like 1.4. The problem is that they only contain discriminants with very few prime factors. For instance, of the 93424 real quadratic discriminants $D<10^{6}$ in $\mathcal{D}$ there are 75912 for which 


\begin{tabular}{|c|c|c|c|c|c|c|c|}
\hline$t$ & $\alpha_{t}(0)$ & $\alpha_{t}(1)$ & $\alpha_{t}(2)$ & $\alpha_{t}(3)$ & $\alpha_{t}(4)$ & $\alpha_{t}(5)$ & $P_{t}$ \\
\hline 1 & 1 & & & & & & 1 \\
\hline 2 & .5 & .5 & & & & & .66666667 \\
\hline 3 & .5 & .375 & . 125 & & & & .64285714 \\
\hline 4 & .4375 & .4375 & 列. & .015625 & & & .60000000 \\
\hline 5 & 4375 & .41015625 & .13671875 & .01464844 & .00097656 & & .59475806 \\
\hline 6 & .42382812 & .42382812 & .13244629 & .01892090 & .00094604 & .00003052 & .58531746 \\
\hline 7 & .42382812 & .41720581 & 13906860 & .01862526 & .00124168 & .00003004 & .58404589 \\
\hline 8 & .42051697 & .42051697 & . 13798213 & .01971173 & .00123198 & .00003974 & .58175551 \\
\hline 9 & .42051697 & .41887432 & .13962477 & .01963473 & .00130898 & .00003959 & .58143998 \\
\hline 10 & .41969565 & .41969565 & .13935207 & .01990744 & .00130643 & .00004214 & .58087161 \\
\hline$:$ & : & ( & : & & & & \\
\hline$\infty$ & .41942244 & .41942244 & .13980748 & .01997250 & .00133150 & .00004295 & .58057756 \\
\hline
\end{tabular}

TABLE 2. Numerical values of $\alpha_{t}(e)$ and $P_{t}$, for $t \leq 10$ and for $t=\infty$, to eight decimal places.

the negative Pell equation is solvable, which gives a fraction .813 that is well above the conjectured value $P$ from 1.5 . However, of these discriminants there are $39176(41.9 \%)$ in $\mathcal{D}_{1}$ and $40271(43.1 \%)$ in $\mathcal{D}_{2}$, with only $12701(13.6 \%)$ in $\mathcal{D}_{3}$ and 1275 $(1.4 \%)$ in $\mathcal{D}_{t}$ for $t>3$. It is therefore not surprising that we do not find an approximation to $P_{\infty}=$ $\lim _{t \rightarrow \infty} P_{t}$, but something close to the weighted average $.419 P_{1}+.431 P_{2}+.136 P_{3}+.014 P_{4}=.802$. In order to find values close to $P_{\infty}$, one would have to count the frequency of $N \varepsilon_{D}=-1$ among discriminants $D \in \mathcal{D}$ in an interval $I$ for which the expected number of primes in $D$ is large. This means that $\log \log D$ has to be large, and such values of $D$ are obviously intractable for a computer. (If $D$ has a million decimal digits, $\log \log D$ is still smaller than 15.)

It is nevertheless possible to use small discriminants only to get a fair impression of the numerical behavior of the Pell equation. We give the behavior for the initial interval $I=\left[1,2 \cdot 10^{7}\right]$ in Table 3 and for $I=\left[10^{10}, 10^{10}+2 \cdot 10^{7}\right]$ in Table 4 .

Our heuristics predict that the ratio $\pi_{t}(e)=$ $\#\left(\mathcal{D}_{t}(e) \cap I\right) / \#\left(\mathcal{D}_{t}^{-}(e) \cap I\right)$ (in the lower right corner of each inner box) should be close to $2^{e+1}-1$, and we have a theorem asserting that the ratio $a_{t}(e)=\#\left(\mathcal{D}_{t}(e) \cap I\right) / \#\left(\mathcal{D}_{t} \cap I\right)$ (upper right corner) tends to $\alpha_{t}(e)$ if the endpoint of the interval tends to infinity. It is clear from these tables that as soon as there are not too few discriminants in the corresponding entry, the numbers $\pi_{t}(e)$ and $\pi(e)=$ $\#(\mathcal{D}(e) \cap I) / \#\left(\mathcal{D}^{-}(e) \cap I\right)$ are reasonably close to their conjectured values. This indicates that the basic assumption of our heuristics works well in practice. On the other hand, the values of $a_{t}(e)$ are sometimes quite far from their known limit values $\alpha_{t}(e)$, and as a consequence $\Pi_{t}=\#\left(\mathcal{D}_{t}^{-} \cap\right.$ $I) / \#\left(\mathcal{D}_{t} \cap I\right)$ differs considerably from its conjectured limit $P_{t}$. This slow convergence is mostly due to the fact that for intervals containing only discriminants of moderate size, the discriminants divisible by certain small primes occur much more frequently than those without these small factors. As an example, look at $t=3$, where many discriminants $D=p_{1} p_{2} p_{3}$ occur with $p_{1} p_{2}$ equal to the 4 smallest possible values $40,65,85$ or 104 . It happens that we have $\left(\frac{p_{1}}{p_{2}}\right)=-1$ in each of these cases, so these discriminants never give $e=2$ and $e=1$ only for $p_{3}$ that satisfy $\left(\frac{p_{1}}{p_{3}}\right)=\left(\frac{p_{2}}{p_{3}}\right)=1$. This explains the inequalities $a_{3}(0)>\alpha_{3}(0)$ and $a_{3}(2)<\alpha_{3}(2)$ in our tables and accounts for the fact that $\Pi_{3}$ is larger than $P_{3}$. Analogous phenomena occur for all values $t \geq 3$. Note however that the effect is already less pronounced in the second of the two tables. The strong influence of these "numerical coincidences" only disappears for large 


\begin{tabular}{|c|c|c|c|c|c|c|c|c|c|c|}
\hline$t$ & \multicolumn{2}{|c|}{$e=0$} & \multicolumn{2}{|c|}{$e=1$} & \multicolumn{2}{|c|}{$e=2$} & \multicolumn{2}{|c|}{$e=3$} & sum & $\Pi_{t}$ \\
\hline 1 & $\begin{array}{l}635171 \\
635171\end{array}$ & $\begin{array}{l}1 \\
1\end{array}$ & & & & & & & $\begin{array}{l}635171 \\
635171\end{array}$ & 1.000 \\
\hline 2 & $\begin{array}{l}367180 \\
367180\end{array}$ & $\begin{array}{r}.5023 \\
1\end{array}$ & $\begin{array}{l}363827 \\
120554\end{array}$ & $\begin{array}{l}.4977 \\
3.018\end{array}$ & & & & & $\begin{array}{l}731007 \\
487734\end{array}$ & .6672 \\
\hline 3 & $\begin{array}{l}160468 \\
160468\end{array}$ & $\begin{array}{r}.5625 \\
1\end{array}$ & $\begin{array}{l}98281 \\
32607\end{array}$ & $\begin{array}{l}.3445 \\
3.014\end{array}$ & $\begin{array}{r}26550 \\
3588\end{array}$ & $\begin{array}{l}.0931 \\
7.400\end{array}$ & & & $\begin{array}{l}285299 \\
196663\end{array}$ & .6893 \\
\hline 4 & $\begin{array}{l}21848 \\
21848\end{array}$ & $\begin{array}{r}.5050 \\
1\end{array}$ & $\begin{array}{r}18563 \\
6061\end{array}$ & $\begin{array}{l}.4291 \\
3.063\end{array}$ & $\begin{array}{r}2756 \\
360\end{array}$ & $\begin{array}{l}.0637 \\
7.656\end{array}$ & $\begin{array}{r}96 \\
5\end{array}$ & $\begin{array}{l}.0022 \\
19.20\end{array}$ & $\begin{array}{l}43263 \\
28274\end{array}$ & .6535 \\
\hline 5 & $\begin{array}{l}1090 \\
1090\end{array}$ & $\begin{array}{r}.5375 \\
1\end{array}$ & $\begin{array}{l}790 \\
266\end{array}$ & $\begin{array}{l}.3895 \\
2.970\end{array}$ & $\begin{array}{r}143 \\
22\end{array}$ & $\begin{array}{l}.0705 \\
6.500\end{array}$ & $\begin{array}{l}5 \\
0\end{array}$ & $\begin{array}{r}.0025 \\
*\end{array}$ & $\begin{array}{l}2028 \\
1378\end{array}$ & .6795 \\
\hline 6 & $\begin{array}{l}6 \\
6\end{array}$ & $\begin{array}{r}.6667 \\
1\end{array}$ & $\begin{array}{l}2 \\
1\end{array}$ & $\begin{array}{l}.2222 \\
2.000\end{array}$ & $\begin{array}{l}1 \\
0\end{array}$ & $\begin{array}{r}.1111 \\
*\end{array}$ & $\begin{array}{l}0 \\
0\end{array}$ & & $\begin{array}{l}9 \\
7\end{array}$ & .7778 \\
\hline sum & $\begin{array}{l}1185763 \\
1185763\end{array}$ & 1 & $\begin{array}{l}481463 \\
159489\end{array}$ & 3.019 & $\begin{array}{r}29450 \\
3970\end{array}$ & 7.418 & $\begin{array}{r}101 \\
5\end{array}$ & 20.20 & $\begin{array}{l}1696777 \\
1349227\end{array}$ & .7952 \\
\hline
\end{tabular}

TABLE 3. Numerical data for $D<2 \cdot 10^{7}$. The upper and lower left numbers in each entry $(t, e)$ count how many values of $D$ in the interval $I=\left[1,2 \cdot 10^{7}\right]$ are in $\mathcal{D}_{t}(e)$ and $\mathcal{D}_{t}^{-}(e)$. Each entry further lists their quotient $\pi_{t}(e)=\#\left(\mathcal{D}_{t}(e) \cap I\right) / \#\left(\mathcal{D}_{t}^{-}(e) \cap I\right)$ in the lower right corner and the fraction $a_{t}(e)=\#\left(\mathcal{D}_{t}(e) \cap I\right) / \#\left(\mathcal{D}_{t} \cap I\right)$ in the upper right corner. The edges of the table give the row and column totals showing the solvability of the Pell equation in $I$ for fixed $t$ and for fixed $e$. For fixed $t$ we list $\Pi_{t}=\#\left(\mathcal{D}_{t}^{-} \cap I\right) / \#\left(\mathcal{D}_{t} \cap I\right)=\sum_{e=0}^{t-1} a_{t}(e) / \pi_{t}(e)$, and for fixed $e$ we list $\pi(e)=\#(\mathcal{D}(e) \cap I) / \#\left(\mathcal{D}^{-}(e) \cap I\right)$. The value of $\Pi_{t}$ is heuristically close to $P_{t}$, and that of $\pi(e)$ to $2^{e+1}-1$.

\begin{tabular}{|c|c|c|c|c|c|c|c|c|c|c|}
\hline$t$ & \multicolumn{2}{|c|}{$e=0$} & \multicolumn{2}{|c|}{$e=1$} & \multicolumn{2}{|c|}{$e=2$} & \multicolumn{2}{|c|}{$e=3$} & sum & $\Pi_{t}$ \\
\hline 1 & $\begin{array}{l}434574 \\
434574\end{array}$ & $\begin{array}{l}1 \\
1\end{array}$ & & & & & & & $\begin{array}{l}434574 \\
434574\end{array}$ & 1.000 \\
\hline 2 & $\begin{array}{l}296434 \\
296434\end{array}$ & $\begin{array}{r}.5001 \\
1\end{array}$ & $\begin{array}{r}296363 \\
98701\end{array}$ & $\begin{array}{l}.4999 \\
3.003\end{array}$ & & & & & $\begin{array}{l}395135 \\
992797\end{array}$ & .6666 \\
\hline 3 & $\begin{array}{l}163853 \\
163853\end{array}$ & $\begin{array}{r}.5328 \\
1\end{array}$ & $\begin{array}{r}110388 \\
36817\end{array}$ & $\begin{array}{l}.3590 \\
2.998\end{array}$ & $\begin{array}{r}33276 \\
4584\end{array}$ & $\begin{array}{l}.1082 \\
7.259\end{array}$ & & & $\begin{array}{l}307517 \\
205254\end{array}$ & .6675 \\
\hline 4 & $\begin{array}{l}35500 \\
35500\end{array}$ & $\begin{array}{r}.4695 \\
1\end{array}$ & $\begin{array}{l}33107 \\
10993\end{array}$ & $\begin{array}{l}.4379 \\
3.012\end{array}$ & $\begin{array}{r}6394 \\
861\end{array}$ & $\begin{array}{l}.0846 \\
7.426\end{array}$ & $\begin{array}{r}610 \\
35\end{array}$ & $\begin{array}{l}.0081 \\
17.42\end{array}$ & $\begin{array}{l}75611 \\
47389\end{array}$ & .6267 \\
\hline 5 & $\begin{array}{l}4390 \\
4390\end{array}$ & $\begin{array}{r}.4796 \\
1\end{array}$ & $\begin{array}{l}3723 \\
1216\end{array}$ & $\begin{array}{l}.4068 \\
3.062\end{array}$ & $\begin{array}{l}983 \\
131\end{array}$ & $\begin{array}{l}.1074 \\
7.504\end{array}$ & $\begin{array}{r}56 \\
2\end{array}$ & $\begin{array}{l}.0061 \\
28.00\end{array}$ & $\begin{array}{l}9153 \\
5739\end{array}$ & .6270 \\
\hline 6 & $\begin{array}{l}230 \\
230\end{array}$ & $\begin{array}{r}.4915 \\
1\end{array}$ & $\begin{array}{r}192 \\
64\end{array}$ & $\begin{array}{l}.4103 \\
3.000\end{array}$ & $\begin{array}{l}43 \\
10\end{array}$ & $\begin{array}{l}.0919 \\
4.300\end{array}$ & $\begin{array}{l}3 \\
0\end{array}$ & $\begin{array}{r}.0064 \\
*\end{array}$ & $\begin{array}{l}468 \\
304\end{array}$ & .6496 \\
\hline 7 & $\begin{array}{l}4 \\
4\end{array}$ & $\begin{array}{r}.5714 \\
1\end{array}$ & $\begin{array}{l}2 \\
1\end{array}$ & $\begin{array}{l}.2857 \\
3.000\end{array}$ & $\begin{array}{l}1 \\
0\end{array}$ & $\begin{array}{r}.1429 \\
*\end{array}$ & & & $\begin{array}{l}7 \\
5\end{array}$ & .7143 \\
\hline sum & $\begin{array}{l}934985 \\
934985\end{array}$ & 1 & $\begin{array}{l}443775 \\
147792\end{array}$ & 3.003 & $\begin{array}{r}40697 \\
5586\end{array}$ & 7.288 & $\begin{array}{r}669 \\
37\end{array}$ & 18.08 & $\begin{array}{l}1420127 \\
1088400\end{array}$ & .7664 \\
\hline
\end{tabular}

TABLE 4. Numerical data for $10^{10}<D<10^{10}+2 \cdot 10^{7}$. The data are organized in the same fashion as in Table 3 , with $I=\left[10^{10}, 10^{10}+2 \cdot 10^{7}\right]$. This table represents a much larger computational effort than Table 3 , and was compiled in cooperation with Wieb Bosma from the Cayley group in Sydney. There is a single discriminant in $\mathcal{D}_{5}(4)$ that is not included here. 
$\log \log D$, and this does not occur in situations that can be handled by a computer. However, we need not worry about the behavior of the fractions $a_{t}(e)$, since we have a theorem telling us what their limit behavior is. The only quantity that is important for our heuristics is $\pi_{t}(e)$, and the tables show that their predicted behavior is well matched by our numerical data.

We observe that, even in the second interval, there are no discriminants with $e>3$ besides the single value

$D=5 \cdot 29 \cdot 241 \cdot 349 \cdot 821=10012755905 \in \mathcal{D}_{5}(4)$

(not shown in Table 4). This is not too surprising, since these discriminants form a very thin set that is contained in $\bigcup_{t>5} \mathcal{D}_{t}$. It is possible to treat such discriminants, but not with the straightforward algorithms used in compiling the preceding tables. For a more sophisticated computational approach in the line of [Lagarias 1980] the reader is referred to a forthcoming paper [Bosma and Stevenhagen]. This paper also examines the more general case of (1.1) in which $d$ is no longer supposed to be squarefree. The (heuristic) analysis of this case requires distribution hypotheses for units in arbitrary quadratic orders that are very different from our basic Hypothesis 3.1 [Stevenhagen b].

We conclude this section with the observation that even if we were able to prove the heuristics concerning $P_{t}$ for all $t$, this still would not imply Conjecture 3.4. Proving the implication involves a change of limits,

$$
\lim _{t \rightarrow \infty} \lim _{X \rightarrow \infty} \frac{\#\left(\mathcal{D}_{t}^{-}\right)_{\leqslant x}}{\#\left(\mathcal{D}_{t}\right)_{\leqslant x}}=\lim _{X \rightarrow \infty} \lim _{t \rightarrow \infty} \frac{\#\left(\mathcal{D}_{t}^{-}\right)_{\leqslant x}}{\#\left(\mathcal{D}_{t}\right)_{\leqslant x}}
$$

which may be hard to justify. As an example showing the difficulties that arise, one can look at the subset of even discriminants in $\mathcal{D}$. This set has positive density in $\mathcal{D}$, but it follows from (2.6) that its intersection with $\mathcal{D}_{t}$ has zero density in $\mathcal{D}_{t}$ for each $t$.

\section{PROVEN DENSITIES FOR THE NEGATIVE PELL EQUATION}

In this section we will see how close we can get to an unconditional proof of Theorem 3.2. More precisely, we will give lower bounds for

$$
\underline{P}_{t}=\liminf _{X \rightarrow \infty} \frac{\#\left(\mathcal{D}_{t}^{-}\right)_{\leqslant X}}{\#\left(\mathcal{D}_{t}\right)_{\leqslant X}}
$$

and upper bounds for

$$
\bar{P}_{t}=\limsup _{X \rightarrow \infty} \frac{\#\left(\mathcal{D}_{t}^{-}\right)_{\leqslant X}}{\#\left(\mathcal{D}_{t}\right)_{\leqslant X}} .
$$

The first result in this direction goes back to Rédei $[1936 ; 1939]$. It is the observation that, for $D \in \mathcal{D}_{t}$, the negative Pell equation is solvable when $e_{4}(D)=$ 0 . This follows immediately from Lemma 2.1(iii), because the narrow 2-Hilbert class field coincides under this assumption with the (real) genus field $G$. Using Proposition 2.2, we can phrase the result as follows:

Proposition 4.1. Let $D \in \mathcal{D}_{t}$ be a discriminant for which the Rédei matrix $M_{D}$ has maximal rank $t-1$. Then $D$ is in $\mathcal{D}_{t}^{-}$.

This result has been rediscovered in various guises by several authors [Cremona and Odoni 1989; Hurrelbrink 1990; Pumplün 1968; Trotter 1969].

Corollary 4.2. Let $\alpha$ be defined as in (2.7). Then, for every $t \geq 1$,

$$
\underline{P}_{t} \geq \alpha_{t}(0)>\alpha_{\infty}(0)=\alpha .
$$

This result suggests that $\alpha$ can be taken as a lower bound for the lower density of $\mathcal{D}^{-}$in $\mathcal{D}$, but for reasons explained at the end of the previous section this is still unproved.

There is a numerical observation in [Cremona and Odoni 1989] that the equivalent of Proposition (4.1) in that paper accounts for about $87 \%$ of all $D$ in $\mathcal{D}^{-}$when squarefree radicands are considered up to $10^{6}$, and the question is raised why the criterion should be so effective. This is explained by our heuristics, which imply that, for fixed $t$, the proposition accounts for a fraction $f_{t}=\alpha_{t}(0) / P_{t}$ of 
all $D$ in $\mathcal{D}_{t}^{-}$. To four decimals, we have $f_{1}=1, f_{2}=$ $.75, f_{3}=.7778, f_{4}=.7292$, and $f_{\infty}=.7218$. Using the distribution over the various $\mathcal{D}_{t}$ for $D<10^{6}$ mentioned in the previous section, one should expect the weighted average $.419 f_{1}+.431 f_{2}+.136 f_{3}+$ $.014 f_{4}$ of these values as the effective percentage for the criterion, and this is about $86 \%$. The numerical values of $f_{i}$ found for the set of discriminants up to $10^{6}$ are $1, .759, .831$ and .790 , giving rise to a percentage of $89 \%$. The slightly higher numerical values for $f_{3}$ and $f_{4}$ are well explained by the "numerical coincidences" discussed in the previous section. In the limit case $D<\infty$, the proposition should account for $\alpha /(1-\alpha)=72.18 \%$ of all $D$ in $\mathcal{D}^{-}$.

Now suppose that $D=p_{1} p_{2} \ldots p_{t} \in \mathcal{D}$ is a discriminant for which $e=e_{4}(D)$ is positive. Then the genus field $G$ of $K=\mathbf{Q}(\sqrt{D})$ is strictly contained in the narrow 2-Hilbert class field of $K$, and the solvability of the negative Pell equation is no longer determined by the values of the Legendre symbols $\left(\frac{p_{i}}{p_{j}}\right)$ only. However, it is possible to determine in terms of the $p_{i}$ the archimedean character of the subfield $F$ of the narrow Hilbert class field of $K$ that corresponds to the fourth powers in the class group $C_{D}$. The field $F$, which is known as the narrow 4-Hilbert class field of $K$, is an extension of the genus field $G$ of degree $2^{e}$ and therefore not abelian over $\mathbf{Q}$.

The two theorems we will use can be seen as generalizations of Dirichlet's biquadratic result mentioned in the introduction and go back to [Rédei 1939] and [Scholz 1935].

Given $D=p_{1} p_{2} \ldots p_{t}$, every nonzero element $v=\left(v_{i}\right)_{i=1}^{t}$ of $V=\mathbf{F}_{2}^{t}$ corresponds to a discriminantal divisor $D_{1}=\prod_{v_{i}=1} p_{i}$ of $D$. Setting $D_{2}=$ $D / D_{1}$, the decomposition $D=D_{1} \cdot D_{2}$ is said to be of the second kind if it comes from an element $v$ in the kernel of the Redei matrix $M_{D}$. In more elementary terms, this means that $D_{1}$ is a square modulo all primes in $D_{2}$ and $D_{2}$ a square modulo all primes in $D_{1}$. Note that the trivial elements 0 and $u=(1)_{i=1}^{t}$ in this kernel give rise to the trivial decompositions $D=1 \cdot D=D \cdot 1$. For every nontrivial decomposition $D=D_{1} \cdot D_{2}$, one can construct a subextension $K \subset M$ of $K \subset F$ that is cyclic of degree 4 and contains $K \subset \mathbf{Q}\left(\sqrt{D_{1}}, \sqrt{D_{2}}\right)$ as a quadratic subextension. The decompositions of the second kind correspond bijectively to the elements of $\operatorname{ker} M_{D}$, so they have a natural vector space structure over $\mathbf{F}_{2}$. By passing to the quotient space $\left(\operatorname{ker} M_{D}\right) /\left(\mathbf{F}_{2} \cdot u\right)$, we can identify decompositions $D=D_{1} \cdot D_{2}$ and $D=D_{2} \cdot D_{1}$. The space thus obtained has dimension $e_{4}(D)$ and is known as the space of decompositions of the second kind.

We can now formulate the first result of Rédei and Scholz. In the special case that the discriminant $D$ is of the form $D=p q \in \mathcal{D}_{2}$ with $\left(\frac{p}{q}\right)=1$, we obtain Dirichlet's result mentioned in the Introduction.

Theorem 4.3. Let $F$ be the narrow 4-Hilbert class field of the real quadratic field of discriminant $D \in$ D. Then $F$ is real if and only if for every decomposition of the second kind $D=D_{1} \cdot D_{2}$, we have

$$
\left(\frac{D_{1}}{D_{2}}\right)_{4}\left(\frac{D_{2}}{D_{1}}\right)_{4}=1
$$

In particular, the negative Pell equation for $D$ can only be solvable if this condition is satisfied.

In the preceding theorem, one can read " $e_{4}(D)$ independent decompositions of the second kind" instead of "every decomposition of the second kind", since the map

$$
D_{1} \cdot D_{2} \mapsto\left(\frac{D_{1}}{D_{2}}\right)_{4}\left(\frac{D_{2}}{D_{1}}\right)_{4}
$$

is a linear functional on the space of decompositions of the second kind. Note that this functional can only be defined for decompositions of the second kind. It can be shown [Rédei 1939] that for the set of discriminants $D$ giving rise to some fixed Rédei matrix, the $e$ independent conditions from Theorem (4.3) are satisfied for a subset of density $2^{-e}$. This implies that the upper density of $\mathcal{D}_{t}^{-}(e)$ in $\mathcal{D}_{t}(e)$ is bounded by $2^{-e}$, and it yields the following upper bound for $\bar{P}_{t}$ : 
Corollary 4.4. For any integer $t \geq 1$, the upper density $\bar{P}_{t}$ satisfies

$$
\bar{P}_{t} \leq \sum_{e \geq 0} 2^{-e} \alpha_{t}(e)
$$

In the limit $t \rightarrow \infty$, this gives $\lim \sup _{t \rightarrow \infty} \bar{P}_{t} \leq \frac{2}{3}$.

Proof. The first statement is clear from the argument preceding the corollary. For the identity $\sum_{e \geq 0} 2^{-e} \alpha_{\infty}(e)=\frac{2}{3}$, it suffices to show that if we let $A=\prod_{j \text { odd }}\left(1-q^{-j}\right)$, then

$$
S=\sum_{e=0}^{\infty} q^{-e} \frac{A}{\prod_{j=1}^{e}\left(q^{j}-1\right)}=\frac{q}{q+1}
$$

for every complex number $q$ with $|q|>1$. To do this one writes

$$
\frac{1}{q^{e}\left(q^{e}-1\right)}=\frac{1}{q^{e}-1}-\frac{1}{q^{e}}
$$

in all terms of $S$ having $e \geq 1$. This yields

$$
\begin{aligned}
S & =A+\sum_{e=1}^{\infty} \frac{A}{\prod_{j=1}^{e}\left(q^{j}-1\right)}-\sum_{e=1}^{\infty} q^{-e} \frac{A}{\prod_{j=1}^{e-1}\left(q^{j}-1\right)} \\
& =1-\frac{S}{q}
\end{aligned}
$$

by (2.9), whence the result.

The argument we gave implies that the negative Pell equation is solvable with a very low probability for discriminants that give rise to high 4-ranks. All density results for special families derived by Morton [1982] apply to discriminants having this property, so it is not surprising that his upper densities are very different from those we find for the full set $\mathcal{D}_{t}$.
For the lower density $\underline{P}_{t}$ we can use the following sufficient condition for solvability of the negative Pell equation, also due to Rédei and Scholz.

Theorem 4.5. Let $D \in \mathcal{D}$ be a real quadratic discriminant, and suppose that

$$
\left(\frac{D_{1}}{D_{2}}\right)_{4}=-1=\left(\frac{D_{2}}{D_{1}}\right)_{4}
$$

for every decomposition $D=D_{1} \cdot D_{2}$ of the second kind. Then we have $e_{4}(D) \leq 2$ and $N \varepsilon_{D}=-1$.

In this case it is not sufficient to have the identities only for a basis of the decompositions of the second kind. For a given decomposition $D=D_{1} \cdot D_{2}$, they imply that there is no cyclic unramified extension $K \subset M$ of degree 8 with $K \subset \mathbf{Q}\left(\sqrt{D_{1}}, \sqrt{D_{2}}\right)$ as its quadratic subextension. It is not hard to see that the identities cannot hold for all 7 nontrivial decompositions generated by 3 independent decompositions of the second kind. This explains why we must have $e_{4}(D) \leq 2$. For $e_{4}(D)=1$ we have two independent conditions, and for $e_{4}(D)=2$ we have five independent conditions as the 6 biquadratic symbols coming from the 3 nontrivial decompositions of the second kind have product 1 . As before, we deduce that the lower density of $\mathcal{D}_{t}^{-}(e)$ in $\mathcal{D}_{t}(e)$ is at least $2^{-2}$ when $e=1$ and at least $2^{-5}$ when $e=2$.

Corollary 4.6. For any integer $t \geq 1$, the lower density $\underline{P}_{t}$ satisfies

$$
\underline{P}_{t} \geq \alpha_{t}(0)+\frac{1}{4} \alpha_{t}(1)+\frac{1}{32} \alpha_{t}(2)
$$

In particular, $\liminf \operatorname{in}_{t \rightarrow \infty} \underline{P}_{t} \geq \frac{121}{96} \alpha=.528647 \ldots$

\begin{tabular}{|c|cccccc|c|}
\hline bounds & $t=2$ & 3 & 4 & 5 & 6 & 7 & $\infty$ \\
\hline $\bar{P}_{t} \leq$ & .75000 & .71875 & .68555 & .67865 & .67128 & .66960 & .66667 \\
$P_{t}$ & .66667 & .64286 & .60000 & .59476 & .58532 & .58405 & .58058 \\
$\underline{P}_{t} \geq$ & .62500 & .59766 & .55029 & .54431 & .53392 & .53248 & .52865 \\
\hline
\end{tabular}

TABLE 5. Proven upper and lower bounds related to $P_{t}$. 
In conclusion, we list in Table 5 the values of the proven lower and upper bounds for $\bar{P}_{t}$ and $\underline{P}_{t}$, together with the heuristic value of $P_{t}$ for $2 \leq t \leq 7$ and for $t=\infty$. (Obviously, $\bar{P}_{1}=\underline{P}_{1}=P_{1}=1$.) In order to improve these bounds, one would need density results like Proposition 2.5 for the higher Rédei matrices defined in [Stevenhagen a].

\section{ACKNOWLEDGEMENTS}

I thank Wieb Bosma for his help in compiling the results in Table 4 and Rob Tijdeman for pointing out that the identity (2.9) implies the irrationality of the conjectural value of $P$ in Conjecture 1.4. Hendrik Lenstra suggested improvements in an earlier version of the manuscript.

\section{REFERENCES}

[Beach and Williams 1972] B. D. Beach and H. C. Williams, "A numerical investigation of the Diophantine equation $x^{2}-d y^{2}=-1$ ", Proc. Southeastern Conf. on Combinatorics, Graph Theory and Computing (1972), 37-52.

[Borevich and Shafarevich 1966] Z. I. Borevich and I. R. Shafarevich, Number theory, Academic Press, New York, 1966.

[Bosma and Stevenhagen] W. Bosma and P. Stevenhagen, "Density computations real quadratic class groups", in preparation.

[Cohen and Lenstra 1984] H. Cohen and H. W. Lenstra, Jr., "Heuristics on class groups", pp. 3362 in Number Theory: Noordwijkerhout 1983, edited by H. Jager, Lecture Notes in Math. 1086, SpringerVerlag, Berlin, 1984.

[Cremona and Odoni 1989] J. E. Cremona and R. W. K. Odoni, "Some density results for negative Pell equations; an application of graph theory", $J$. London Math. Soc. (2) 39 (1989), 16-28.

[Dirichlet 1834] P. G. L. Dirichlet, "Einige neue Sätze über unbestimmte Gleichungen", Abh. K. Preuss. Akad. d. Wiss. (1834), 649-664. Also pp. 219-236 in G. Lejeune-Dirichlet's Werke, Band I, Reimer, Berlin, 1889, reprinted by Chelsea, Bronx (NY), 1969.
[Gerth 1984] F. Gerth III, "The 4-class ranks of quadratic fields", Inv. Math. 77 (1984), 489-515.

[Hardy and Wright 1979] G. H. Hardy and E. M. Wright, An Introduction to the Theory of Numbers, 5th ed., Oxford University Press, Oxford, 1979.

[Hurrelbrink 1990] J. Hurrelbrink, "On the norm of the fundamental unit" (preprint), Louisiana State University, Baton Rouge, 1990.

[Lagarias 1980] J. C. Lagarias, "On the computational complexity of determining the solvability or unsolvability of the equation $X^{2}-D Y^{2}=-1$ ", Trans. Amer. Math. Soc. 260 (1980), 485-508.

[MacWilliams 1969] J. MacWilliams, "Orthogonal matrices over finite fields", Amer. Math. Monthly $\mathbf{7 6}$ (1969), 152-164.

[Morton 1979] P. Morton, "On Rédei's theory of the Pell equation", J. reine angew. Math. 307/308 (1979), 373-398.

[Morton 1982] P. Morton, "Density results for the 2classgroups and fundamental units of real quadratic fields", Studia Sci. Math. Hungar. 17 (1982), 21-43.

[Nagell 1932] T. Nagell, "Über die Lösbarkeit der Gleichung $x^{2}-D y^{2}=-1$ ", Arkiv för Mat. Astr. Fysik 23 B/6 (1932), 1-5.

[R. V. Perlis 1990] R. V. Perlis, "On the density of fields with $N(\varepsilon)=-1$ " (preprint), Louisiana State University, Baton Rouge, 1990.

[Pumplün 1968] D. Pumplün, "Über die Klassenzahl und die Grundeinheit des reell-quadratischen Zahlkörpers", J. reine angew. Math. 230 (1968), 167-210.

[Rédei and Reichardt 1934] L. Rédei and H. Reichardt, "Die Anzahl der durch 4 teilbaren Invarianten der Klassengruppe eines beliebigen quadratischen Zahlkörpers", J. reine angew. Math. 170 (1934), 69-74.

[Rédei 1935a] L. Rédei, "Über die Grundeinheit und die durch 8 teilbaren Invarianten der absoluten Klassengruppe im quadratischen Zahlkörper", $J$. reine angew. Math. 171 (1935), 131-148.

[Rédei 1935b] L. Rédei, "Über die Pellsche Gleichung $t^{2}-d u^{2}=-1 "$, J. reine angew. Math. 173 (1935), 193-221. 
[Rédei 1936] L. Rédei, "Über einige Mittelwertfragen im quadratischen Zahlkörper", J. reine angew. Math. 174 (1936), 131-148.

[Rédei 1939] L. Rédei, "Ein neues zahlentheoretisches Symbol mit Anwendungen auf die Theorie der quadratischen Zahlkörper", J. reine angew. Math. 180 (1939), 1-43.

[Rédei 1953a] L. Rédei, "Bedingtes Artinsches Symbol mit Anwendungen in der Klassenkörpertheorie", Acta Math. Hungar. 4 (1953), 1-29.

[Rédei 1953b] L. Rédei, "Die 2-Ringklassengruppe des quadratischen Zahlkörpers und die Theorie der Pellschen Gleichung", Acta Math. Hungar. 4 (1953), 31-87.

[Rieger 1965] G. J. Rieger, "Über die Anzahl der als Summe von zwei Quadraten darstellbaren und in einer primen Restklasse gelegenen Zahlen unterhalb einer positiven Schranke. II", J. reine angew. Math. 217 (1965), 200-216.
[Scholz 1935] A. Scholz, "Über die Lösbarkeit der Gleichung $t^{2}-D u^{2}=-4$ ", Math. Z. 39 (1935), 95111.

[Stark 1970] H. M. Stark, An Introduction to Number Theory, MIT Press, Cambridge (MA), 1970.

[Stevenhagen 1993] P. Stevenhagen, "On the 2-power divisibility of certain quadratic class numbers", $J$. Number Theory 43(1) (1993), 1-19.

[Stevenhagen a] P. Stevenhagen, "Rédei matrices and applications", Séminaire de Théorie des Nombres Paris 1991-92 (to appear).

[Stevenhagen b] P. Stevenhagen, "A density conjecture for the negative Pell equation", Proc. Conf. Algebra and Number Theory, Sydney 1992 (to appear).

[Trotter 1969] H. F. Trotter, "On the norm of units in quadratic fields", Proc. Amer. Math. Soc. 22 (1969), 198-201.

[Thielmann 1926] M. von Thielmann, "Zur Pellschen Gleichung", Math. Ann. 95 (1926), 635-640.

[Weil 1984] A. Weil, Number Theory: An Approach through History, Birkhäuser, Boston, 1984.

Peter Stevenhagen, Faculteit Wiskunde en Informatica, Plantage Muidergracht 24, 1018 TV Amsterdam, Netherlands (psh@fwi.uva.nl)

Received June 15, 1993; accepted July 23 